\title{
Report of the Tobacco Policy Research Study Group on Adjunctive Medications for Managing Nicotine Dependence
}

\author{
John Slade, Gregory N Connolly, Ronald M Davis, Clifford E Douglas, Jack E \\ Henningfield, John R Hughes, Lynn T Kozlowski, Matthew L Myers
}

\begin{abstract}
Introduction
Because smoking is so common, and because its complications are so common and so devastating, ${ }^{1}$ nicotine dependence is the most common serious medical problem in the United States. ${ }^{2,3}$ Clinical approaches to management of this condition have improved markedly over the past 15 years. ${ }^{3,4}$ Substantial advances in behavioural therapy have been complemented by the introduction of pharmacological agents that ameliorate nicotine withdrawal. Though these major advances have been enormously useful, substantial research is needed to advance basic knowledge of treatment, to integrate what has already been learnt into daily practice for individuals and in the community, and to inform policies that foster the availability of appropriate treatment where it is needed most. This report focuses on the unanswered policy research questions about using pharmacological adjuncts in managing nicotine dependence.
\end{abstract}

\section{Massachusetts Department of Public Health, Boston, Massachusetts G N Connolly}

Michigan Department of Public Health, Lansing, Michigan R M Davis

Advocacy Institute, Washington, DC C E Douglas

National Institute on Drug Abuse,

Baltimore, Maryland

$\mathrm{J}$ E Henningfield

University of Vermont, Burlington, Vermont

J R Hughes

Pennsylvania State University, University Park, Pennsylvania L T Kozlowski

Asbill, Junkin, and Myers, Chtd, and Coalition on Smoking or Health or Health,
Washington, DC M L Myers

Correspondence to: Dr John Slade, Department of Medicine, St Peter's Medical Center, 254 Easton Medical Center, 254 Easton Avenue, PO Box 591, N Brunswick, New Jers

\section{Background} ket and promote nicotine in several forms, as well as drugs similar to nicotine, as adjuncts in the treatment of nicotine dependence. The goal in this arena is the familiar one of producing a safe and effective drug. ${ }^{4-6}$ Nicotine gum (Nicorette, available in the United States since 1984), various nicotine patches (Habitrol, Nicoderm, Prostep, and Nicotrol, first introduced in the United States in late 1991), and lobeline-containing preparations sold over the counter (such as Ban-Tron, Cigarrest, and Nicoban) are the nicotinic agonists presently on the market for this indication. Nicotine gum and nicotine patches are effective as adjuncts in the behavioural treatment of nicotine dependence. On the other hand, although lobeline has possible peripheral nicotinic actions, activity in the central nervous system has not been shown, and products containing lobeline have no demonstrated efficacy. Other formulations containing nicotine (nasal sprays and vapour inhalers) are under development. In the United States both nicotine gum and nicotine patches are available only on prescription, whereas lobeline-containing products are sold over the counter.

Physicians serve as gatekeepers for nicotine gum and patches since these are prescription
A number of pharmaceutical companies mar- items. Theoretically, this provides people who smoke with a setting in which to receive counselling and behavioural therapy, or referral for these services, along with a prescription. Limiting access with prescriptions also reduces diversion for unapproved uses that can actually sustain continued tobacco use, such as using a patch when it is inconvenient to smoke.

Conventional pharmaceutical development and clinical research procedures have been and are being used to ascertain the degree to which a given form of nicotine is safe and effective in the management of nicotine dependence. Nicotine gum and nicotine patches are safe and effective in helping people stop smoking when used as adjuncts to behavioural therapies for nicotine dependence. In fact, the Food and Drug Administration (FDA) has approved these drugs for use only in the context of a behavioural treatment programme for the purpose of stopping smoking. (Approval for a specific indication such as this means that the manufacturer can promote a drug only for an approved purpose. FDA approval does not limit a practitioner from prescribing a drug for another, "off label" use.)

These drugs are not very useful as treatments on their own, however. ${ }^{7}$ This is not surprising: medicines alone, without behavioural or other non-pharmacological treatment or support, have never been strikingly effective in the management of other chemical dependencies such as alcoholism and heroin addiction.

Over $95 \%$ of smokers who used nicotine gum did not participate in behavioural therapy or counselling. ${ }^{8}$ Patch manufacturers have been criticised for advertising that emphasises nicotine replacement and de-emphasises the need for concomitant behavioural treatment of the addiction, ${ }^{9}$ and the FDA has recently indicated its interest in postmarketing surveillance studies of the extent to which people receiving prescriptions for patches also receive counselling or behavioural therapy. ${ }^{10}$

Unfortunately, the clinical research establishing the effectiveness of replacement agents such as nicotine gum and patches does not address all of the relevant policy issues raised by these drugs. The data addressing these problems are scant. Five of the issues raised are described below. 
DIVERSION FROM INTENDED USE

As nicotine regularly produces a chemical dependence, preparations containing nicotine or similar drugs intended for use in treating nicotine dependence may be diverted from their labelled indication to help sustain tobacco use. For instance, nicotine gum has been used as a temporary substitute for cigarettes when it is inconvenient or impossible to smoke. This use is not necessarily bad (for instance, when a hospital inpatient is made more comfortable with nicotine gum even though the person has no plan to stop smoking or, perhaps, when an airline pilot is not allowed to smoke in the cockpit during flight), but it has the potential of making it less likely that an individual will try to stop smoking (for instance, a person who would try to stop smoking as a result of workplace restrictions on smoking but for the availability of nicotine gum to minimise discomfort during working hours).

These considerations are similar to the ways methadone is regarded in the management of heroin withdrawal among people not intending to stop opiate use. Hospitalised patients and medically supervised outpatients are offered pharmacological support for detoxification, while the use of "street methadone" to bridge between adequate heroin supplies is regarded as a form of drug abuse which contributes to continued, active opiate dependence.

\section{REFRACTORINESS TO STOPPING SMOKING} AMONG PEOPLE WHO RELAPSE

Most people who try to stop smoking relapse. However, continued effort increases the chances of success. Thus, increasing the likelihood that people who have relapsed do not become discouraged and refractory to further attempts to stop should be a major goal for both clinical management and public health practice.

Present understanding of this phenomenon is rudimentary, but aspects of the problem can be discerned from what smokers report about previous, unsuccessful attempts to stop smoking. Smokers report feeling discouraged by relapses, seemingly in proportion to the time, energy, and money they have invested in the process. Unsuccessful treatments linked to the medical community, especially treatments involving the dispensing of drugs, may be especially powerful inducers of discouragement among people who relapse or who fail to stop in the first place.

However, the smoker is often unaware that the treatment itself may have been defective. For instance, nicotine gum is approved for use in behavioural treatments. However, the dispensers of nicotine gum are usually not trained to offer the basic behavioural treatment, and the providers who offer behavioural programmes are usually not reimbursed by third party payers. ${ }^{11}$ An effect of unsuccessful quitting after the use of nicotine gum or other organised, formal approaches might be a period of relative refractoriness to trying to stop again. If this occurs, this refractoriness might be seen as an adverse effect of the drug or other treatment. The degree to which this is a clinically important problem and the ways it might be mitigated are fruitful topics for research.

\section{NICOTINE MAINTENANCE}

Although nicotine gum is approved only for use as a temporary adjunct for people trying to become abstinent, a few patients have found it extremely difficult to stop using the gum. ${ }^{12}$ In some people depressive symptoms develop with attempts to stop smoking, and this condition can often be alleviated with a return to smoking or with antidepressant drugs. ${ }^{13}$ (People with a history of depression or who have high depression symptom scores are at increased risk of smoking and have a more difficult time stopping.) The extent to which difficulty in stopping taking nicotine gum represents a deep attachment to the behaviours associated with smoking, self medication with nicotine for depression, or a result of more or less permanent biological responses to years of nicotine ingestion is unclear.

The percentage of people receiving nicotine replacement who have difficulty stopping all nicotine ingestion is relatively small (about $20 \%$ of those who stop smoking with nicotine gum). None the less, this represents a very large number of people. It seems appropriate to explore a maintenance rather than a detoxification strategy for at least some of these people. This is feasible to consider because nicotine by itself seems to have an acceptable safety profile for selected patients compared with drugs accepted for use in long term treatments of other, equally or even less serious diseases. ${ }^{4,14}$ In short, research is needed which would inform policies about making genuinely safe alternative products available to people who continue to use nicotine while continuing to encourage abstinence as the primary approach.

\section{REFINING CLINICAL PRACTICE}

Once a drug is approved and marketed there is little incentive for a manufacturer to sponsor studies of more precise dosing and duration of treatment, effects on specific subpopulations, new indications, cost-benefit analyses of various management strategies, and the like. Relevant questions in this category include both optimal techniques for individual patient care and public health planning.

It would be helpful to know if there are differences among various groups in the optimal dosing of nicotine preparations. That this might be the case is suggested by differences in the average numbers of cigarettes consumed by white Americans and AfricanAmericans, alcoholics and non-alcoholics, etc. Particular attention should be focused on the optimal management of pregnant and lactating women who continue to smoke despite intense social pressure. These women are at high risk of complications because of tobacco use. They are also more available to intervention through prenatal and postnatal medi- 
cal care than are many other population groups. Importantly, cessation of smoking early in pregnancy completely reverses prenatal risk. ${ }^{15}$ Concern about weight gain is an important clinical issue for many women who consider stopping smoking. Optimal approaches to this issue continue to be refined as understanding of the problem advances. ${ }^{16}$

These and many other related questions are important in the clinical management of nicotine dependence. Ways to routinely extend clinical research beyond that which is necessary for initial approval by the FDA should be developed for this class of agent.

UNDERSERVED POPULATIONS

One specific concern is the likely maldistribution of treatment in relation to need. Although smokers are increasingly concentrated in low income groups, services to help people stop smoking are more readily available in affluent areas. ${ }^{17}$

\section{Research priorities}

There are several critical areas for research on drugs to treat nicotine addiction. These include postmarketing studies, studies of alternative availability for existing products, exploration of nicotine maintenance, refinement of clinical practice, and the targeting of treatment for underserved populations.

The existing research and drug development infrastructure is sufficient to support the development of useful drugs for the management of nicotine dependence. Additional research approaches are needed, however, to explore important public health and clinical issues which conventional drug development does not address. Five of these issues are described below.

\section{POSTMARKETING SURVEILLANCE}

Questions to be addressed include:

- What treatment, advice, and recommendations are given by physicians in conjunction with a prescription for nicotine gum or patch?

- What do people who smoke do after being offered behavioural treatment and an adjunctive drug? How are the treatment and the drug used?

- To what extent is the prescribed drug used to supplement conventional tobacco consumption when it is inconvenient or impossible to smoke?

- What happens to people who try to stop smoking but who relapse? Is there a refractory period in which the person is less likely to try to stop? Does the method used in a particular attempt at stopping affect the duration or the intensity of any refractory period before the next attempt?

- What is the abuse liability of various nicotine replacement products? Are they attractive to people who have not smoked or used other tobacco products? Is their use associated with difficulty stopping or with continued use of tobacco products? Are former smokers attracted to them, creating the potential that these products represent a new route for relapse to smoking? Nicotine gum and patches have been regarded as having low abuse liability because of their formulations, but there is substantial reason for concern with the nicotine nose sprays and inhalers currently under development, because they more closely resemble commercial tobacco products in their pharmacokinetics.

\section{ALTERNATIVE MARKETING STRATEGIES}

Nicotine gum is already sold over the counter in several places, including Great Britain, Switzerland, Argentina, Australia, and Hong Kong. Studies of its usefulness in helping people stop smoking at various levels of availability (requiring a visit to a physician attracts a different population than being able to buy a preparation over the counter) and its potential diversion as a temporary substitute for smoking should be conducted in these places. The results of such studies would form part of an evaluation of whether nicotine replacement products should be available over the counter in the United States and, if so, how they should be marketed.

\section{NICOTINE MAINTENANCE}

Nicotine dependence has an enormously broad clinical spectrum. There are both various degrees of dependence and various intercurrent clinical problems (such as depression and alcohol dependence) which interfere with the achievement of abstinence. The cultural environment is another important variable as well, and as this changes towards offering greater support for abstinence, many people find it easier to stop.

Studies are needed to better characterise the variations in severity of nicotine dependence and to explore the potential need for a maintenance treatment for selected subgroups. Several experienced clinicians believe that, as long as tobacco products are available, many users will never become abstinent from nicotine. It is therefore appropriate to explore ways that nicotine might be offered as maintenance treatment.

Such a research programme must consist of six basic elements:

- Epidemiology of the severity of nicotine dependence and coexistent illnesses that interfere with recovery

- Drug development including clinical trials to identify the least hazardous, acceptable nicotinic agonists

- Research on the long term effects of nicotine alone on human health such as comparing the health benefits which accrue to those who stop consuming tobacco with long term use of nicotine replacement and those who become completely abstinent from nicotine

- Comparisons of the risk of relapse to tobacco use among those abstinent from nicotine and those who continue to take nicotine as a maintenance drug 
- Comparison of products that deliver nicotine intermittently or for 12-16 hours daily with those that deliver nicotine continuously, around the clock

- Design of a clinically acceptable service delivery system to make the drug available to those otherwise unable to abstain from tobacco products as well as limit availability to others.

All six elements are needed for there to be enough information to decide whether such an approach is feasible and would make a useful contribution to public health.

REFINING CLINICAL PRACTICE

Who uses treatment services, including adjunctive drugs, to help stop smoking? Is use more concentrated among better educated, more affluent people? People with lower incomes and educational attainment are more likely to be more highly dependent on nicotine and so have a larger potential benefit from these drugs. What can be done to facilitate these drugs being made available to underserved populations?

Pharmaceutical companies conduct clinical trials of an experimental drug as part of the process for obtaining approval from the FDA for marketing the drug. Once approval is obtained, the incentive for conducting further studies is markedly reduced. At the same time, an ongoing commitment to clinical investigation of adjunctive therapies for nicotine dependence beyond that which is necessary for initial approval is important to advance clinical practice. Optimal doses and duration of treatment, tailoring treatment to particular subsets of patients, and combining various drugs and other modalities are some of the topics of importance in refining clinical practices.

\section{REACHING UNDERSERVED POPULATIONS}

Who uses treatment services, including adjunctive drugs, to help stop smoking? Is use more concentrated among better educated, more affluent people? People with lower incomes and educational attainment are more likely to be more highly dependent on nicotine and so receive a larger potential benefit from these drugs. What can be done to facilitate these drugs being made available to underserved populations?

\section{Conclusions}

Adjunctive drugs for managing nicotine dependence are important therapeutic advances. Their approved use, however, is only as a support in the treatment of nicotine dependence, and there are several ways these agents may be misused or abused. Unfortunately, the extent of these problems has not been well documented. Agents under development, including nicotine inhalers and nasal sprays, have a greater potential for use in ways which actually sustain tobacco consumption and which pose a risk of use by new initiates.

A small number of people may be unable to stop using nicotine. Nicotine maintenance strategies should be developed and systematically studied for this refractory population.

Clinical practice raises numerous practical questions about optimal treatment for nicotine dependence, and there should be an organised, ongoing effort to address these issues. The distribution of services for nicotine dependence may be inversely related to need. ${ }^{17}$ This issue should be explored and better characterised.

Nicotine gum and nicotine patches have been important additions to the other clinical tools for helping people stop smoking. There are ways these drugs can hinder as well as help, however, and clinicians have only begun to characterise the optimal role these drugs should play in the management of nicotine dependence.

1 Peto R, Lopez AD, Boreham J, Thun M, Heath C Jr Mortality from tobacco in developed countries: indirect estimation from national vital statistics. Lancet 1992 339: $1268-78$

2 Henningfield JE, Cohen C, Slade J. Is nicotine mor addictive than cocaine? $\mathrm{Br} \mathcal{F}$ Addict 1991; 86: 565-9.

3 US Department of Health and Human Services. Reducing the health consequences of smoking : 25 years of progress. $A$ report of the Surgeon General, 1989. Atlant progress. A report of the Surgeon General, 1989. Atlanta, Georgia Centers for Disease Control, Office on Smoking and

4 US Department of Health and Human Services. The health consequences of smoking : nicotine addiction. A report of the Surgeon General, 1988. Atlanta, Georgia: Centers fo Surgeon General, 1988. Atlanta, Georgia: Centers for
Disease Control, Office on Smoking and Health, 1988. (DHHS Publication No (CDC) 88-8406.)

5 Henningfield JR, Stitzer ML, eds. New developments in nicotine delivery systems. New York: Carlton, 1991.

6 Jarvik ME, Henningfield JE. Pharmacological treatment of tobacco dependence. Pharmacol Biochem Behav 1988; 30 : 379-94

7 Hughes JR. Problems of nicotine gum. In: Pomerleau OF, Pomerleau C, Fagerstrom K-O, eds. Nicotine replacement in the treatment of smoking. New York: Plenum Press, 1988: 261-78.

8 Hughes JR. Problems of nicotine gum. In: Ockene JK, ed. The pharmacologic treatment of tobacco dependence: proceedings of the world congress November 4-5, 1985. Cambridge, Massachusetts: Institute for the Study of Smoking Behavior and Policy, 1986.

9 Wolfe S, Sharfstein JM, Slade J. Petition to the Food and Drug Administration to require certain additional warnings on advertising for nicotine patches. Public Citizen 1992 Apr 30. (Washington, DC.)

10 Food and Drug Administration. Letter from Bruce $\mathrm{V}$ Stadel, Chief, Epidemiology Branch, Division of Epidemiology and Surveillance, Office of Epidemiology and Biostatistics, to John Slade, Robert Wood Johnson Medical School (New Brunswick, New Jersey) 24 March 1992.

11 Parkinson MD, Schauffler $\mathrm{HH}$, Kottke TE, Curry SJ, Solberg LI, Arnold CB, et al. Report of the Tobacco Policy Research Study Group on Reimbursement and Insurance in the United States. Tobacco Control 1992; 1 (suppl): S2-S6.

12 Hughes JR, Gust SW, Keenan R, Fenwick JW, Skoog K, Higgins ST. Long-term use of nicotine vs placebo gum. Arch Intern Med 1991; 151 : 1993-8.

13 Covey LS, Glassman AH, Stetner F. Depression and depressive symptoms in smoking cessation. Compr Psychiatry 1990; 31: 350-4.

14 Hughes JR. Risk-benefit of nicotine preparations in smoking cessation. Drug Safety (in press)

15 US Department of Health and Human Services. The health consequences of smoking for women. Washington, DC. Public Health Service, Office of the Assistant Secretary for Health, Office on Smoking and Health, 1980.

16 Williamson DF, Madans J, Anda RF, Kleinman JC Giovino GA, Byers T. Smoking cessation and severity of weight gain in a national cohort. $N$ Engl f Med 1991; 324 : 739-45.

17 Caplan RL, Greenberg M, Bellet L. For-profit smokingcessation programs and their clients. Nf Med $1989 ; 86$ $181-3$. 